\title{
Simple efficient bounds for arcsine and arctangent functions
}

\section{Yogesh J. Bagul ( $\nabla$ yjbagul@gmail.com )}

K. K. M. Collge, Manwath, Dist: Parbhani(M. S.)-431505, India https://orcid.org/0000-0002-8331-3920

\section{Ramkrishna M. Dhaigude}

Government Vidarbha Institute of Science and Humanities, Amravati(M. S.)-444604, India

\section{Research Article}

Keywords: Shafer's inequality, Shafer-Fink's inequality, arcsine function, arctangent function, approximate integral.

Posted Date: April 9th, 2021

DOI: https://doi.org/10.21203/rs.3.rs-404784/v1

License: (c) (1) This work is licensed under a Creative Commons Attribution 4.0 International License. Read Full License 


\title{
Simple efficient bounds for arcsine and arctangent functions
}

\author{
Yogesh J. Bagul ${ }^{1, *}$ \\ Department of Mathematics, \\ K. K. M. College, Manwath, \\ Dist: Parbhani-431505, Maharashtra, India. \\ Email: yjbagul@gmail.com \\ Ramkrishna M. Dhaigude ${ }^{2}$ \\ Department of Mathematics, \\ Government Vidarbha Institute of Science and Humanities, \\ Amravati-444604,Maharashtra, India. \\ Email : rmdhaigude@gmail.com
}

\begin{abstract}
The aim of this paper is to present new, simple and sufficiently sharp bounds for arcsine and arctangent functions. Some of the bounds are computationally efficient while others are efficient to approximate the integrals $\int_{a}^{b} \frac{\arcsin x}{x} d x$ and $\int_{a}^{b} \frac{\arctan x}{x} d x$. As a matter of interest, several other sharp and generalized inequalities for $\frac{\arcsin x}{x}$ and $\frac{\arctan x}{x}$ are also established which are efficient to give some known and other trigonometric inequalities
\end{abstract}

AMS(MOS) Subject Classification Codes: 26D05, 26D20, 42A10. Keywords: Shafer's inequality, Shafer-Fink's inequality, arcsine function, arctangent function, approximate integral.

\section{INTRODUCTION}

The inequalities

$$
\frac{\arcsin x}{x}<\frac{1}{\sqrt{1-x^{2}}} ; x \in(0,1)
$$

and

$$
\frac{1}{1+x^{2}}<\frac{\arctan x}{x} ; x>0
$$

are easy consequences of Mean Value Theorem(MVT) [8]. These inequalities can be found in many textbooks of undergraduate course.

${ }^{*}$ Corresponding author 
They are also often taken as an application of "Meaning of sign of derivative". For example, the above inequalities follow since the derivatives of the functions $x-\sqrt{1-x^{2}} \arcsin x$ and $\left(1+x^{2}\right) \arctan x-x$ are clearly positive. The inequality (1.1) is sharp towards the origin, whereas inequality (1.2) is sharp as $x \rightarrow 0$ and $x \rightarrow \infty$. The importance of these inequalities lies in their simplicity. i.e., they contain less number of arithmetic/non-arithmetic operations. In the literature there are also other bounds for $\frac{\arcsin x}{x}$ and $\frac{\arctan x}{x}$. They are cited as below.

The Shafer-Fink's inequality $[10,18]$ states that

$$
\frac{3}{2+\sqrt{1-x^{2}}}<\frac{\arcsin x}{x}<\frac{\pi}{2+\sqrt{1-x^{2}}} ; x \in(0,1) .
$$

L. Zhu [26] improved double inequality (1.3) to

$$
\Omega(x)<\frac{\arcsin x}{x}<\frac{\pi}{2+(\pi-2) \sqrt{1-x^{2}}} ; x \in(0,1),
$$

where $\Omega(x)=\Omega_{1}(x)$ or $\Omega_{2}(x)$ and $\Omega_{1}(x)=\frac{\pi(4-\pi)}{2 /(\pi-2)+\sqrt{1-x^{2}}}$, and $\Omega_{2}(x)=$ $\frac{\pi}{2+2 \sqrt{1-x^{2}}}$. Shafer [23] in 1966, proposed the inequality

$$
\frac{3}{1+2 \sqrt{1+x^{2}}}<\frac{\arctan x}{x} ; x>0
$$

as a problem. Further, in 1977-78, Shafer [24,25] obtained the following inequality:

$$
\frac{8}{3+\sqrt{25+\frac{80}{3} x^{2}}}<\frac{\arctan x}{x} ; x>0 .
$$

The extension of (1.6) to

$$
\frac{8}{3+\sqrt{25+\frac{80}{3} x^{2}}}<\frac{\arctan x}{x}<\frac{8}{3+\sqrt{25+\frac{256}{\pi^{2}} x^{2}}} ; x>0
$$

was obtained in [27] by L. Zhu. Recently the inequalities (1.3)-(1.7) have been sharpened by many researchers(see for instance, $[9,11,15$, 16, 19, 20,27] and references therein). Though the bounds for $\frac{\arcsin x}{x}$ and $\frac{\arctan x}{x}$ in (1.3)-(1.7) or in recent papers $[9,11,15,16,19,20,27]$ are sharp they are not computationally as efficient as the bounds in (1.1) and (1.2), in the sense of number of arithmetic/non-arithmetic 
operations involved in them(see [21]). According to [21] any function is computationally efficient if it contains less number of arithmetic and non-arithmetic operations. In this context, among all the bounds mentioned so far in this paper, including those in references $[9,11,15,16,19,20,27]$ (in fact, the bounds given in these references contain more operations), the bounds in (1.1) and (1.2) are most computationally efficient.

Secondly, consider the integral $\int_{a}^{b} f(x) d x$, provided that we can find anti-derivative $F$ such that $F^{\prime}(x)=f(x)$ where $x \in[a, b]$. Then by Fundamental Theorem of Calculus [8], we can easily evaluate the integral $\int_{a}^{b} f(x) d x$. However, when we can not find such an $F$, we are not able to use Fundamental Theorem of Calculus. In this situation, we find bounds $g$ and $h$ which are accurate enough for our needs such that $g \leqslant f \leqslant h$ and the integrals of $g$ and $h$ are calculated easily. So $\left(\int_{a}^{b} g(x) d x+\int_{a}^{b} h(x) d x\right) / 2$ is the approximate integral of $\int_{a}^{b} f(x) d x$. This is elementary procedure to find quick estimate of $\int_{a}^{b} f(x) d x$. Now it is not difficult to observe that the recent bounds mentioned above are not useful for the quick estimates of $\int_{a}^{b} \frac{\arcsin x}{x} d x$ and $\int_{a}^{b} \frac{\arctan x}{x} d x$. (it is to be noted however that those recent bounds are better to find quick estimates of $\int_{a}^{b} \frac{x}{\arcsin x} d x$ and $\left.\int_{a}^{b} \frac{x}{\arctan x} d x\right)$.

In this paper, we will obtain inequalities for $\frac{\arcsin x}{x}$ and $\frac{\arctan x}{x}$ in such a way that some of our established bounds are computationally efficient while others are efficient to approximate $\int_{a}^{b} \frac{\arcsin x}{x} d x$ and $\int_{a}^{b} \frac{\arctan x}{x} d x$. In addition to this, as a matter of interest, we will also establish several generalized inequalities for $\frac{\arcsin x}{x}$ and $\frac{\arctan x}{x}$. These generalized inequalities will give us some known and other trigonometric inequalities.

\section{Results And Discussion}

In this section, we propose our main results. The first one is stated as follows.

Proposition 1. Let $x \in(0,1)$. Then

$$
1+\frac{x^{2}}{6}<\frac{\arcsin x}{x}<1+\frac{(\pi-2)}{2} x^{2}
$$


The lower bound in (2.1) is more sharp as compared to it's upper bound. Both these bounds are sharpened in Proposition 2.

Proposition 2. Let $x \in(0,1)$. Then

$$
\frac{6}{6-x^{2}}<\frac{\arcsin x}{x}<\frac{\pi}{\pi-(\pi-1) x^{2}} .
$$

Bounds in (2.1) and (2.2) are sharper than the corresponding one in (1.1). These bounds in (2.1) and (2.2) can be used to approximate the integral $\int_{0}^{1} \frac{\arcsin x}{x} d x$. For getting better approximation we have bounds in the following theorem.

Theorem 1. Let $x \in(0,1)$. Then the inequalities

$$
\frac{1}{\sqrt{1-\frac{x^{2}}{3}}}<\frac{\arcsin x}{x}<\frac{1}{\sqrt{1-\left(\frac{\pi^{2}-4}{\pi^{2}}\right) x^{2}}}
$$

hold.

In the following, very simple bounds for $\frac{\arctan x}{x}$ are proposed.

Proposition 3. For $x>0$ it is asserted that

$$
\frac{3}{3+x^{2}}<\frac{\arctan x}{x}<\frac{4}{3+\theta x},
$$

where 1 and $\theta=2.06341 \cdots$ are the best possible constants.

Thus the inequality (1.2) is sharpened and extended in (2.4). For better bounds we have

Theorem 2. For $x>0$, the inequalities

$$
\frac{1}{\sqrt{1+\frac{2}{3} x^{2}}}<\frac{\arctan x}{x}<\frac{1}{\sqrt{1+\frac{4}{\pi^{2}} x^{2}}}
$$

hold.

We have other bounds for $\frac{\arctan x}{x}$ quite similar to those in (2.4). They are presented in the next proposition.

Proposition 4. For $x>0$ it is asserted that

$$
\frac{\pi}{\pi+2 x}<\frac{\arctan x}{x}<\frac{3}{2+\vartheta x},
$$

where 2 and $\vartheta=1.70895 \cdots$ are the best possible constants. 
Remark 1. The lower bound of (2.6) is sharper than the corresponding lower bound of (2.5) except for $x \in\left(0,6 \pi /\left(\pi^{2}-6\right)\right)$ and it is also sharper than the corresponding lower bound of (2.4) except for $x \in(0,6 / \pi)$. Similarly the upper bound of (2.6) is sharper than the corresponding upper bound of (2.4) except for $x \in(0,1.549019 .$.$) .$

As a matter of interest, we establish several inequalities in the coming theorems.

Theorem 3. Let $x \in(0,1)$ and $a \in(0,1]$. Then the function $f(x)=$ $\frac{\ln \left(\frac{\arcsin x}{x}\right)}{\ln \left(1-a x^{2}\right)}$ is strictly decreasing if $a \leqslant 1 / 2$. In particular, we have following several inequalities

$$
\frac{1}{\left(1-a x^{2}\right)^{\alpha}}<\frac{\arcsin x}{x}<\frac{1}{\left(1-a x^{2}\right)^{\beta}},
$$

with the best possible constants $\alpha=\frac{1}{6 a}$ and $\beta=\frac{\ln (2 / \pi)}{\ln (1-a)}$.

Putting $a=1 / 3$ in (2.7) we get

$$
\frac{1}{\sqrt{1-\frac{x^{2}}{3}}}<\frac{\arcsin x}{x}<\frac{1}{\left(1-\frac{x^{2}}{3}\right)^{\beta_{1}}},
$$

where $\beta_{1}=\frac{\ln (2 / \pi)}{\ln (2 / 3)} \approx 1.113739$. Similarly, putting $a=1 / 2$ in (2.7) we get

$$
\frac{1}{\left(1-\frac{x^{2}}{2}\right)^{1 / 3}}<\frac{\arcsin x}{x}<\frac{1}{\left(1-\frac{x^{2}}{2}\right)^{\beta_{2}}},
$$

where $\beta_{2}=\frac{\ln (2 / \pi)}{\ln (1 / 2)} \approx 0.651496$.

Inequalities in (2.9) are sharpest inequalities of kind (2.7). The lower bound in (2.9) is better than corresponding lower bounds in (1.4), though there is no strict comparison with $\Omega_{1}(x)$. Similarly the upper bound in (2.9) is sharper in major portion of $(0,1)$ than the corresponding upper bound in (1.3), particularly as $x \rightarrow 0$, this upper bound is very much better than the one in (1.3).

Our last result is about generalized bounds for $\frac{\arctan x}{x}$.

Theorem 4. Let $x \in(0, \lambda)$ where $\lambda \in(0, \infty)$. Then the function $g(x)=$ $\frac{\ln \left(\frac{x}{\arctan x}\right)}{\ln \left(1+a x^{2}\right)}$ is strictly increasing if $a \geqslant 1$. In particular, we have following several inequalities 


$$
\frac{1}{\left(1+a x^{2}\right)^{\eta}}<\frac{\arctan x}{x}<\frac{1}{\left(1+a x^{2}\right)^{\xi}}
$$

with the best possible constants $\eta=\frac{\ln \left(\frac{\lambda}{\arctan \lambda}\right)}{\ln \left(1+a \lambda^{2}\right)}$ and $\xi=\frac{1}{3 a}$.

Since $\eta \rightarrow \frac{1}{2}$ as $\lambda \rightarrow \infty$, the inequalities (2.10) can be rewritten as

$$
\frac{1}{\sqrt{1+a x^{2}}}<\frac{\arctan x}{x}<\frac{1}{\left(1+a x^{2}\right)^{1 / 3 a}} ; x>0 .
$$

The sharpest inequalities of the kind (2.11) are observed at $a=1$ which are given below.

$$
\frac{1}{\sqrt{1+x^{2}}}<\frac{\arctan x}{x}<\frac{1}{\left(1+x^{2}\right)^{1 / 3}} ; x>0 .
$$

The proofs of these results are based on secondary lemmas which are presented in the next section.

\section{Five Lemmas}

Following power series expansions can be found in [27, Lemma 1].

Lemma 1. Let $|t|<\pi$. Then

$$
\cot ^{2} t=\frac{1}{t^{2}}-\frac{2}{3}+\sum_{n=2}^{\infty} \frac{2^{2 n}}{(2 n) !}(2 n-1)\left|B_{2 n}\right| t^{2 n-2},
$$

and

$$
\frac{1}{\sin ^{2} t}=\frac{1}{t^{2}}+\sum_{n=1}^{\infty} \frac{2^{2 n}}{(2 n) !}(2 n-1)\left|B_{2 n}\right| t^{2 n-2} .
$$

where $B_{2 n}$ are the even-indexed Bernoulli numbers.

Lemma 2 [1, p.10] taken below is known as l'Hôpital's rule of monotonicity. It is indispensable tool in the theory of inequalities.

Lemma 2. Let $f, g:[m, n] \rightarrow \mathbb{R}$ be two continuous functions which are differentiable on $(m, n)$ and $g^{\prime} \neq 0$ in $(m, n)$. If $f^{\prime} / g^{\prime}$ is increasing (or decreasing) on $(m, n)$, then the functions $\frac{f(x)-f(m)}{g(x)-g(m)}$ and $\frac{f(x)-f(n)}{g(x)-g(n)}$ are also increasing (or decreasing) on $(m, n)$. If $f^{\prime} / g^{\prime}$ is strictly monotone, then the monotonicity in the conclusion is also strict. 
Lemma 3. [17, Theorem 2.1] Let $f:(0, c) \rightarrow \mathbb{R}$ be $m$ times differentiable function ( for some $m \geq 2, m \in \mathbb{N}$ ) which satisfies the following conditions:

(a) $f^{m}(x)>0$ for $x \in(0, c)$;

(b) there is a right neighbourhood of zero in which the following inequalities hold true:

$$
f(x)<0, f^{\prime}(x)<0, \cdots f^{(m-1)}(x)<0
$$

(c) there is a left neighbourhood of $c$ in which the following inequalities hold true:

$$
f(x)>0, f^{\prime}(x)>0, \cdots f^{(m-1)}(x)>0 .
$$

Then the function $f$ has exactly one zero in $(0, c)$.

The following lemma is recently proved by Y. J. Bagul et al. in [7, Lemma 2.2]

Lemma 4. The function $\phi(x)=\frac{x-\sqrt{1-x^{2}} \arcsin x}{x^{2} \arcsin x}$ is positive increasing on $(0,1)$.

In addition to this, we need another lemma that is stated and proved below.

Lemma 5. The function $\psi(x)=\frac{\left(1+x^{2}\right) \arctan x-x}{x^{2} \arctan x}$ is strictly positive increasing for $x>0$.

Proof. Note that $\psi(x)$ is positive due inequality (1.2). Consider

$$
\psi(x)=\frac{\psi_{1}(x)}{\psi_{2}(x)},
$$

where $\psi_{1}(x)=\left(1+x^{2}\right) \arctan x-x$ and $\psi_{2}(x)=x^{2} \arctan x$, satisfying $\psi_{1}(0)=0$ and $\psi_{2}(0)=0$. After differentiating

$$
\frac{\psi_{1}^{\prime}(x)}{\psi_{2}^{\prime}(x)}=\frac{1}{1+\frac{x}{2\left(1+x^{2}\right) \arctan x}}=\frac{1}{1+\frac{1}{2} \psi_{3}(x)} .
$$

Here $\psi_{3}(x)=\frac{\psi_{4}(x)}{\psi_{5}(x)}$ is such that $\psi_{4}(0)=0$ and $\psi_{5}(0)=0$. Differentiation yields

$$
\frac{\psi_{4}^{\prime}(x)}{\psi_{5}^{\prime}(x)}=\frac{1}{1+2 x \arctan x}
$$

which is strictly decreasing as $x$ and $\arctan x$ are strictly increasing for $x>0$. By Lemma $2, \psi_{3}(x)$ is strictly decreasing, whence $\psi(x)$ is strictly increasing for $x>0$. This completes the proof. 


\section{Proofs of main Results}

Proof of Proposition 1. Set

$$
h(x)=\frac{\arcsin x-x}{x^{3}}=\frac{h_{1}(x)}{h_{2}(x)},
$$

where $h_{1}(x)=\arcsin x-x$ and $h_{2}(x)=x^{3}$ with $h_{1}(0)=0$ and $h_{2}(0)=0$. Upon differentiation

$$
\frac{h_{1}^{\prime}(x)}{h_{2}^{\prime}(x)}=\frac{1-\sqrt{1-x^{2}}}{3 x^{2} \sqrt{1-x^{2}}}=\frac{h_{3}(x)}{h_{4}(x)}
$$

so that $h_{3}(0)=0$ and $h_{4}(0)=0$. Again differentiating

$$
\frac{h_{3}^{\prime}(x)}{h_{4}^{\prime}(x)}=\frac{1}{6-9 x^{2}}
$$

which is strictly increasing on $(0,1)$. By Lemma $2, h(x)$ is also strictly increasing on $(0,1)$. Hence

$$
h\left(0^{+}\right)<h(x)<h\left(1^{-}\right) .
$$

The limits $h\left(0^{+}\right)=1 / 6$ and $h\left(1^{-}\right)=(\pi-2) / 2$ finish the proof.

Proof of Proposition 2. Let

$$
K(x)=\frac{\arcsin x-x}{x^{2} \arcsin x}
$$

and

$$
\arcsin x=t \text { i.e., } x=\sin t \text { with } t \in(0, \pi / 2) \text {. }
$$

Then

$$
K(x)=\kappa(t) \text { where } \kappa(t)=\frac{t-\sin t}{t \sin ^{2} t}=\frac{t-\sin t}{t^{2} \sin t} \frac{t}{\sin t}=\kappa_{1}(t) \frac{t}{\sin t} .
$$

Now

$$
\kappa_{1}(t)=\frac{t-\sin t}{t^{2} \sin t} \text { and } \kappa_{1}^{\prime}(t)=2 t \sin ^{2} t-t^{2} \sin t-t^{3} \cos t .
$$

It has second derivative

$$
\begin{aligned}
\kappa_{1}^{\prime \prime}(t) & =(\sin t-t)(4 t \cos t+2 \sin t)+t^{3} \sin t \\
& >(\sin t-t)(4 \sin t+2 \sin t)+t^{3} \sin t
\end{aligned}
$$

by virtue of $t \cos t<\sin t$ and $\sin t<t$. Thereby

$$
\kappa_{1}^{\prime \prime}(t)>\sin t\left(6 \sin t-6 t+t^{3}\right)=\sin t \kappa_{2}(t) .
$$


The derivatives up to second order of $\kappa_{2}$ are

$$
\kappa_{2}^{\prime}(t)=6 \cos t-6+3 t^{2}
$$

and

$$
\kappa_{2}^{\prime \prime}(t)=6(t-\sin t)>0 .
$$

This implies that $\kappa_{2}(t)>0$ which in turn indicates $\kappa_{1}^{\prime \prime}(t)>0$ and $\kappa_{1}^{\prime}(t)>0$. Thus $\kappa_{1}(t)$ is positive increasing, implying that $\kappa(t)$ is increasing on $(0, \pi / 2)$ or $K(x)$ increasing on $(0,1)$. Hence

$$
K\left(0^{+}\right)<K(x)<K\left(1^{-}\right) .
$$

The limits $K\left(0^{+}\right)=\kappa\left(0^{+}\right)=1 / 6$ and $K\left(1^{-}\right)=\kappa\left(\pi / 2^{-}\right)=\pi /(\pi-1)$ give the desired result.

Proof of Theorem 1. Let $F(x)=\frac{1-\left(\frac{x}{\arcsin x}\right)^{2}}{x^{2}}$ and $\arcsin x=t$. Then $x=\sin t$ for $t \in(0, \pi / 2)$ and we have $F(x)=G(t)$ where

$$
G(t)=\frac{1-\left(\frac{\sin t}{t}\right)^{2}}{\sin ^{2} t}=\frac{1}{\sin ^{2} t}-\frac{1}{t^{2}} .
$$

Using (3.2) of Lemma 1, we get

$$
G(t)=\sum_{n=1}^{\infty} \frac{2^{2 n}}{(2 n) !}(2 n-1)\left|B_{2 n}\right| t^{2 n-2} .
$$

It has derivative

$$
G^{\prime}(t)=\sum_{n=1}^{\infty} \frac{2^{2 n}}{(2 n) !}(2 n-1)(2 n-2)\left|B_{2 n}\right| t^{2 n-3}>0 .
$$

We conclude that $G(t)$ is increasing in $(0, \pi / 2)$ or that $F(x)$ is increasing in $(0,1)$. So

$$
F\left(0^{+}\right)<F(x)<F\left(1^{-}\right) .
$$

Lastly the limits $F\left(0^{+}\right)=G\left(0^{+}\right)=1 / 3$ by l'Hôpital's rule and $F\left(1^{-}\right)=$ $G\left(\pi / 2^{-}\right)=\left(\pi^{2}-4\right) / \pi^{2}$ prove our assertion.

Proof of Proposition 3. For the left inequality of (2.4), consider the function

$$
p(x)=\frac{x-\arctan x}{x^{2} \arctan x}=\frac{p_{1}(x)}{p_{2}(x)},
$$


where $p_{1}(x)=x-\arctan x$ and $p_{2}(x)=x^{2} \arctan x$ with $p_{1}(0)=0$ and $p_{2}(0)=0$. Differentiating numerator and denominator yields

$$
\frac{p_{1}^{\prime}(x)}{p_{2}^{\prime}(x)}=\frac{1}{1+2\left(1+x^{2}\right) \frac{\arctan x}{x}}=\frac{1}{1+2 \frac{p_{3}(x)}{p_{4}(x)}},
$$

where $p_{3}(x)=\left(1+x^{2}\right) \arctan x$ and $p_{4}(x)=x$ with $p_{3}(0)=0$ and $p_{4}(0)=0$. Again differentiating we get

$$
\frac{p_{3}^{\prime}(x)}{p_{4}^{\prime}(x)}=1+2 x \arctan x
$$

which is strictly increasing for $x>0$. Applying Lemma 2, we conclude that $p(x)$ is strictly decreasing for $x>0$. Limit $f\left(0^{+}\right)=1 / 3$, give the required left inequality. For the right inequality of (2.4), we use second derivative test. Let

$$
q(x)=\frac{4}{\arctan x}-\frac{3}{x} ; x>0
$$

We differentiate $q(x)$ with respect to $x$ to obtain

$$
\begin{gathered}
q^{\prime}(x)=\frac{3}{x^{2}}-\frac{4}{\left(1+x^{2}\right)(\arctan x)^{2}}, \\
q^{\prime \prime}(x)=\frac{-6}{x^{3}}+\frac{8 x}{\left(\left(1+x^{2}\right) \arctan x\right)^{2}}+\frac{8}{\left(1+x^{2}\right)^{2}(\arctan x)^{3}} .
\end{gathered}
$$

Now let $\arctan x=t$. Then $x=\tan t$ for $t \in(0, \pi / 2)$ and $q^{\prime}(x)=Q(t)$ where

$$
\begin{aligned}
& Q(t)=\frac{3}{\tan ^{2} t}-\frac{4}{\left(1+\tan ^{2} t\right) t^{2}}=\frac{3 \cos ^{2} t}{\sin ^{2} t}-\frac{4 \cos ^{2} t}{t^{2}} . \\
& \text { i.e. } Q(t)=\frac{\varphi(t)}{t^{2} \tan ^{2} t} \text { with } \varphi(t)=4 \cos ^{2} t+3 t^{2}-4
\end{aligned}
$$

We have derivatives of $\varphi$ up to third order as follows:

and

$$
\begin{gathered}
\varphi^{\prime}(t)=-4 \sin 2 t+6 t=-2 t+\frac{16}{3} t^{3}+O\left(t^{5}\right) \\
\varphi^{\prime \prime}(t)=-8 \cos 2 t+6=-2+16 t^{2}-\frac{16}{3} t^{4}+O\left(t^{6}\right)
\end{gathered}
$$

$$
\varphi^{\prime \prime \prime}(t)=16 \sin 2 t>0 .
$$

Thus all the conditions of Lemma 3 are satisfied by $\varphi$ with $m=3$ and hence we conclude that $Q(t)$ has exactly one zero in $(0, \pi / 2)$. By using the bisection method [2] we find that $0.915582 \cdots$ is the unique solution of $Q(t)=0$ in $(0, \pi / 2)$ or equivalently $\nu=1.301296 \cdots$ is the unique 
solution of $q^{\prime}(x)=0$ in $(0, \infty)$. Similarly $q^{\prime \prime}(\nu)=0.425887 \cdots>0$ indicates that $q$ has minimum value at $\nu$ Hence $q(\nu)<q(x)$ and $\theta=q(\nu)=2.06341 \cdots$ The proof is complete.

Proof of Theorem 2. Let $H(x)=\frac{\left(\frac{x}{\arctan x}\right)^{2}-1}{x^{2}}$ and $\arctan x=t$. Then $x=\tan t$ for $t \in(0, \pi / 2)$ and $H(x)=J(t)$ where

$$
J(t)=\frac{\left(\frac{\tan t}{t}\right)^{2}-1}{\tan ^{2} t}=\frac{1}{t^{2}}-\cot ^{2} t
$$

Using (3.1) of Lemma 1, we get

$$
J(t)=\frac{2}{3}-\sum_{n=2}^{\infty} \frac{2^{2 n}}{(2 n) !}(2 n-1)\left|B_{2 n}\right| t^{2 n-2} .
$$

The derivative

$$
J^{\prime}(t)=-\sum_{n=2}^{\infty} \frac{2^{2 n}}{(2 n) !}(2 n-1)(2 n-2)\left|B_{2 n}\right| t^{2 n-3}<0,
$$

implies that $J(t)$ is decreasing on $(0, \pi / 2)$, or that $H(x)$ is decreasing on $(0, \infty)$. Consequently, we have

$$
H\left(0^{+}\right)>H(x)>\lim _{x \rightarrow \infty} H(x) .
$$

Since $H\left(0^{+}\right)=J\left(0^{+}\right)=2 / 3$ and $\lim _{x \rightarrow \infty} H(x)=J\left(\pi / 2^{-}\right)=4 / \pi^{2}$, we get inequalities $(2.7)$.

Proof of Proposition 4. We skip the proof as it is similar to the proof of Proposition 3.

Proof of Theorem 3. Consider

$$
f(x)=\frac{\ln \left(\frac{\arcsin x}{x}\right)}{\ln \left(1-a x^{2}\right)}=\frac{f_{1}(x)}{f_{2}(x)}
$$

where $f_{1}(x)=\ln (\arcsin x / x)$ and $f_{2}(x)=\ln \left(1-a x^{2}\right)$ with $f_{1}\left(0^{+}\right)=0$ and $f_{2}(0)=0$. On differentiating

$$
\frac{f_{1}^{\prime}(x)}{f_{2}^{\prime}(x)}=\frac{1}{2 a} \frac{\left(a x^{2}-1\right)}{\sqrt{1-x^{2}}} \frac{\left(x-\sqrt{1-x^{2}} \arcsin x\right)}{x^{2} \arcsin x}=\frac{1}{2 a} f_{3}(x) \phi(x) .
$$

By Lemma $4, \phi(x)$ is strictly positive increasing and $f_{3}(x)=\left(a x^{2}-\right.$ $1) / \sqrt{1-x^{2}}$ is clearly negative. So by Lemma $2, f(x)$ will be strictly 
decreasing if $f_{3}(x)$ is strictly decreasing in $(0,1)$. i.e.,

$$
f_{3}^{\prime}(x)=2 a x \sqrt{1-x^{2}}+\frac{x\left(a x^{2}-1\right)}{\sqrt{1-x^{2}}}<0 .
$$

Equivalently, $2 a-a x^{2}-1<0$ or $a<\frac{1}{2-x^{2}}=f_{4}(x)$. Thus

$$
a \leqslant \inf \left\{f_{4}(x): x \in(0,1)\right\}=1 / 2 .
$$

The limits $f\left(0^{+}\right)=-1 / 6 a$ and $f\left(1^{-}\right)=\ln (\pi / 2) / \ln (1-a)$ complete the proof.

Proof of Theorem 4. Without loss of generality we take $a>0$. Consider

$$
g(x)=\frac{\ln \left(\frac{x}{\arctan x}\right)}{\ln \left(1+a x^{2}\right)}=\frac{g_{1}(x)}{g_{2}(x)}
$$

where $g_{1}(x)=\ln (x / \arctan x)$ and $g_{2}(x)=\ln \left(1+a x^{2}\right)$ with $g_{1}\left(0^{+}\right)=0$ and $g_{2}(0)=0$. We differentiate numerator and denominator to obtain

$$
\frac{g_{1}^{\prime}(x)}{g_{2}^{\prime}(x)}=\frac{1}{2 a} \frac{\left(1+a x^{2}\right)}{\left(1+x^{2}\right)} \frac{\left(\left(1+x^{2}\right) \arctan x-x\right)}{x^{2} \arctan x}=\frac{1}{2 a} g_{3}(x) \psi(x) .
$$

By Lemma 5, $\psi(x)$ is strictly positive increasing and $g_{3}(x)=(1+$ $\left.a x^{2}\right) /\left(1+x^{2}\right)$ is also clearly positive as $a>0$. Therefore, by Lemma 2 , $g(x)$ will be strictly increasing if $g_{3}(x)$ is increasing. So

$$
g_{3}^{\prime}(x)=2 a x\left(1+x^{2}\right)-2 x\left(1+a x^{2}\right) \geqslant 0 .
$$

Equivalently, $2 a-2 \geqslant 0$ or $a \geqslant 1$. The required inequalities follow due to limits $g\left(0^{+}\right)=1 / 3 a$ and $g\left(\lambda^{-}\right)=\ln (\lambda / \arctan \lambda) / \ln \left(1+a \lambda^{2}\right)$.

Note. Many of the inequalities in our main results can be proved by forming appropriated mixed trigonometric polynomial functions based on methods and algorithms developed in [13] and [14].

\section{Applications}

5.1. Approximate integrals. In this section, we find approximate integrals of $\frac{\arcsin x}{x}$ and $\frac{\arctan x}{x}$. We can utilize inequalities (2.1), (2.2), (2.3), (2.5) since the anti-derivatives of bounds in these inequalities are known functions. 
Example 1. For finding approximation of $\int_{0}^{1} \frac{\arcsin x}{x} d x$, we use (2.3). The inequalities (2.3) give

$$
I_{1}=\int_{0}^{1} \frac{1}{\sqrt{1-\frac{x^{2}}{3}}} d x<\int_{0}^{1} \frac{\arcsin x}{x} d x<I_{2}=\int_{0}^{1} \frac{1}{\sqrt{1-\left(\frac{\pi^{2}-4}{\pi^{2}}\right) x^{2}}} d x .
$$

Now

$$
I_{1}=\sqrt{3}\left[\arcsin \left(\frac{x}{\sqrt{3}}\right)\right]_{0}^{1}=\sqrt{3} \arcsin \left(\frac{1}{\sqrt{3}}\right) \approx 1.066042
$$

and

$$
\begin{aligned}
I_{2}=\frac{\pi}{\sqrt{\pi^{2}-4}}\left[\arcsin \left(\frac{\sqrt{\pi^{2}-4}}{\pi} x\right)\right]_{0}^{1} & =\frac{\pi}{\sqrt{\pi^{2}-4}} \arcsin \left(\frac{\sqrt{\pi^{2}-4}}{\pi}\right) \\
& \approx 1.142005
\end{aligned}
$$

Hence

$$
\int_{0}^{1} \frac{\arcsin x}{x} d x \approx \frac{I_{1}+I_{2}}{2} \approx 1.104023
$$

Computer gives 1.088793 with absolute error $<0.00013$ for the exact integral.

Example 2. We find approximation of $\int_{0}^{10} \frac{\arctan x}{x} d x$ by utilizing inequality (2.5). The inequalities (2.5) yield

$$
I_{1}=\int_{0}^{10} \frac{1}{\sqrt{1+\frac{2}{3} x^{2}}} d x<\int_{0}^{10} \frac{\arctan x}{x} d x<I_{2}=\int_{0}^{10} \frac{1}{\sqrt{1+\frac{4}{\pi^{2}} x^{2}}} d x
$$

$I_{1}$ and $I_{2}$ can be evaluated as

$$
\begin{aligned}
I_{1}=\sqrt{\frac{3}{2}}\left[\ln \left(x+\sqrt{x^{2}+3 / 2}\right)\right]_{0}^{10} & =\sqrt{\frac{3}{2}}[\ln (10+\sqrt{203 / 2})-\ln (\sqrt{3 / 2})] \\
& \approx 3.425279 \\
I_{2}=\frac{\pi}{2}\left[\ln \left(x+\sqrt{x^{2}+\pi^{2} / 4}\right)\right]_{0}^{10} & =\frac{\pi}{2}\left[\ln \left(10+\sqrt{\frac{\pi^{2}+400}{4}}\right)-\ln (\pi / 2)\right] \\
& \approx 4.005942 .
\end{aligned}
$$

Hence

$$
\int_{0}^{10} \frac{\arctan x}{x} d x \approx \frac{I_{1}+I_{2}}{2} \approx 3.71561
$$


Remark 2. Inequalities in Propositions 1, 2, 3 and 4 can also be used to approximate the integrals $\int_{a}^{b} \frac{\arcsin x}{x} d x$ and $\int_{a}^{b} \frac{\arctan x}{x} d x$ as well as the integrals $\int_{a}^{b} \frac{x}{\arcsin x} d x$ and $\int_{a}^{b} \frac{x}{\arctan x} d x$.

5.2. Some inequalities. We would like to bring it to the notice of readers that if we substitute $\arcsin x=t$ in (1.3) and change the variable $t$ to $x$ again, we get following double inequality

$$
\frac{2+\cos x}{\pi}<\frac{\sin x}{x}<\frac{2+\cos x}{3} ; x \in(0, \pi / 2) \text {. }
$$

The right inequality in (5.1) is known in the literature as Cusa-Huygens inequality [12]. We refer readers to [3,4,6] and references therein for detailed information and recent development regarding Cusa-Huygens inequality. It was extended to (5.1) by J. Sándor and R. Oláh-Gál in 2012 [22, Theorem 1]. By similar technique we obtain from (1.1), the inequality $\cos x<\frac{\sin x}{x}$ and from (1.2) the inequality $\frac{\sin x}{x}<\frac{1}{\cos x}$. Combining them we have well known inequality [18]

$$
\cos x<\frac{\sin x}{x}<\frac{1}{\cos x} ; x \in(0, \pi / 2) .
$$

On the other hand (2.12) gives

$$
(\cos x)^{1 / 3}<\frac{\sin x}{x}<1 ; x \in(0, \pi / 2) .
$$

The left inequality in (5.3) is the famous Adamović-Mitrinović inequality(see [18]). Also from (2.8) and (2.9) we derive respectively the inequalities

$$
\left(\frac{2+\cos ^{2} x}{3}\right)^{\beta_{1}}<\frac{\sin x}{x}<\left(\frac{2+\cos ^{2} x}{3}\right)^{1 / 2} ; x \in(0, \pi / 2)
$$

and

$$
\left(\frac{1+\cos ^{2} x}{2}\right)^{\beta_{2}}<\frac{\sin x}{x}<\left(\frac{1+\cos ^{2} x}{2}\right)^{1 / 3} ; x \in(0, \pi / 2)
$$

with $\beta_{1}=\frac{\ln (2 / \pi)}{\ln (2 / 3)} \approx 1.113739$ and $\beta_{2}=\frac{\ln (2 / \pi)}{\ln (1 / 2)} \approx 0.651496$. Inequalities in (5.5) are better than the corresponding inequalities in (5.4) in terms of sharpness. A more sharpened form of (5.4) and (5.5)can be seen in $[3,5]$ which is given below.

$$
\left(\frac{2+\cos x}{3}\right)^{\beta_{1}}<\frac{\sin x}{x}<\left(\frac{2+\cos x}{3}\right) ; x \in(0, \pi / 2)
$$


where $\beta_{1}=\frac{\ln (2 / \pi)}{\ln (2 / 3)} \approx 1.113739$. Now employing the same method of substitution we easily obtain from (5.6) the double inequality

$$
\frac{3}{2+\sqrt{1-x^{2}}}<\frac{\arcsin x}{x}<\left(\frac{3}{2+\sqrt{1-x^{2}}}\right)^{\beta_{1}}
$$

where $\beta_{1}=\frac{\ln (2 / \pi)}{\ln (2 / 3)} \approx 1.113739$. Interestingly inequality (5.7) sharpens the one in (1.3).

\section{Conclusion}

We investigated and obtained some computationally efficient bounds for $\frac{\arcsin x}{x}$ and $\frac{\arctan x}{x}$, see for example inequalities (1.1), (1.2), (2.1), (2.4) and (2.6). Some of our obtained inequalities e.g., (2.2), (2.3), (2.4), (2.5) and (2.6) are efficient for approximating the integrals $\int_{a}^{b} \frac{\arcsin x}{x} d x$ and $\int_{a}^{b} \frac{\arctan x}{x} d x$. These inequalities can also be employed to approximate $\int_{a}^{b} \frac{x}{\arcsin x} d x$ and $\int_{a}^{b} \frac{x}{\arctan x} d x$. We also established generalized inequalities (2.7), (2.10) that give some known and other trigonometric inequalities.

Conflict of interest. The authors declare that they have no conflict of interest.

\section{REFERENCES}

[1] G. D. Anderson, M. K. Vamanamurthy, and M. Vuorinen, Conformal Invariants, Inequalities and Quasiconformal Maps, John Wiley and Sons, New York, 1997.

[2] K. E. Atkinson, An introduction to numerical analysis, John Wiley and sons, New York, 2nd edn., 1991.

[3] Y. J. Bagul, Remark on the paper of Zheng Jie Sun and Ling Zhu, J. Math. Ineqal., Vol. 13, No. 3, pp. 801-803, 2019. Doi: 10.7153/jmi-2019-13-55

[4] Y. J. Bagul, and C. Chesneau, Refined forms of Oppenheim and Cusa-Huygens type inequalities, Acta Comment. Univ. Tartu. Math., Vol. 24, No. 2, pp. 183194, 2020. Doi: 10.12097/ACUTM.2020.24.12

[5] Y. J. Bagul, and C. Chesneau, Some sharp circular and hyperbolic bounds of $\exp \left(-x^{2}\right)$ with applications, Appl. Anal. and Discrete Math., Vol. 14, No. 1, pp. 239-254, 2020.

[6] Y. J. Bagul, and C. Chesneau, and M. Kostić On the Cusa-Huygens inequality, RACSAM Rev. R. Acad. Cienc. Exactas Fís. Nat. Ser. A Mat., Vol. 115, No. 1(29), 2021. Doi: 10.1007/s13398-020-00978-1

[7] Y. J. Bagul, S. B. Thool, and R. M. Dhaigude, On new inequalities involving circular, inverse circular, inverse hyperbolic and exponential functions, Ganita, Vol. 70, No. 2, pp. 67-72, 2020. 
[8] R. G. Bartle, and D. R. Sherbert, Introduction to Real Analysis, John Wiley and Sons(Asia), Singapore, Third ed., 2007.

[9] G. Bercu, Sharp refinements for the inverse sine function related to Shafer-Fink's inequality, Math. Probl. Eng. 2017, 2017:9237932. Doi: 10.1155/2017/9237932

[10] A. M. Fink, Two inequalities, Univ. Beograd. Pub. Elektrotehn. Fak. Ser. Mat. 6(1995), pp. 48-49, 1995.

[11] B.-N. Guo, Q.-M. Luo, and F. Qi, Sharpening and generalizations of ShaferFink's double inequality for the arc sine function, Filomat 27:2(2013), pp. 261265, 2013. Doi: 10.2298/FIL1302261G

[12] C. Huygens, Oeuvres completes, Société Hollondaise des Sciences, Haga, 18881940.

[13] T. Lutovac, B. Malešević, and C. Mortici, The natural algorithmic approach of mixed trigonometric-polynomial problems, J. Inequal. Appl. Vol. 2017, 2017:116, pp. 1-16, 2017. Doi: 10.1186/s13660-017-1392-1

[14] B. Malešević, and M. Makragić, A method for proving some inequalities on mixed trigonometric polynomial functions, J. Math. Ineqal., Vol. 10, No. 3, pp. 849-876, 2016. Doi: 10.7153/jmi-10-69

[15] B. Malešević, M. Rašajski, and T. Lutovac, Refined estimates and generalizations of inequalities related to arctangent function and Shafer's inequality, Mathematical Problems in Engineering, Vol. 2018, Article ID: 4178629, 8 pp., 2018. Doi: $10.1155 / 2018 / 4178629$

[16] B. Malešević, M. Rašajski, and T. Lutovac, Refinements and generalizations of some inequalities of Shafer-Fink's type for the inverse sine function, J. of Inequal. Appl., Vol. 2017, 2017:275, 2017. Doi: 10.1186/s13660-017-1554-1

[17] B. Malešević, T. Lutovac, and B. Banjac, One method for proving some classes of exponential analytical inequalities, Vol. 32, No. 20, pp. 6921-6925, 2018. Doi: 10.2298/FIL1820921M

[18] D. S. Mitrinović, Analytic Inequalities, Springer-Verlag, Berlin, 1970.

[19] Y. Nishizawa, Refined quadratic estimations of Shafer's inequality, Journal of Inequalities and Applications, 2017, 2017:40. Doi: 10.1186/s13660-017-1312-4

[20] Q.-X. Qiao, and C. P. Chen, Approximations to inverse tangent function, Journal of Inequalities and Applications, 2018:141, 2018. Doi: 10.1186/s13660-0181734-7

[21] C. Ramirez, R. Sanchez, V. Kreinovich, and M. Argaez, $\sqrt{x^{2}+\mu}$ is the most computationally efficient smooth approximation to $|x|$ : a proof, Journal of Uncertain Systems, Vol. 8, No. 3, pp. 205-210, 2014.

[22] J. Sándor and R. Oláh-Gal, On Cusa-Huygens type trigonometric and hyperbolic inequalities, Acta. Univ. Sapientiae Mathematica, Vol. 4, No. 2, pp. 145153, 2012.

[23] R. E. Shafer, Elementary Problems: E 1867, The American Mathematical Monthly, Vol. 73, No. 3, p. 309, 1966.

[24] R. E. Shafer, Analytic inequalities obtained by quadratic approximation, Univ. Beograd. Publ. Elektrotehn. Fak. Ser. Mat. Fiz., No. 577-No. 598(1977), pp. 96-97, 1977. 
[25] R. E. Shafer, On quadratic approximation II, Univ. Beograd. Publ. Elektrotehn. Fak. Ser. Mat. Fiz., No. 602-No. 603(1978), pp. 163-170, 1978.

[26] L. Zhu, A solution of a problem of Oppenheim, Math. Inequal. Appl. Vol. 10, No. 1, pp. 57-61, 2007. Doi: 10.7153/mia-10-07

[27] L. Zhu, On a quadratic estimate of Shafer, J. Math. Ineqal., Vol. 2, No. 4, pp. 571-574, 2008. Doi: 10.7153/jmi-02-51 